\title{
Stereotactic Body Radiation Therapy for Treatment of Spinal Bone Metastasis
}

\author{
Yasemin Benderli Cihan
}

\begin{abstract}
Stereotactic body radiation therapy (SBRT) appears an effective and safe treatment modality for spinal bone metastasis, which can enhance local control and improve quality of life. Life expectation, predicted fracture risk, localization, quality, size and number of metastasis and presence or absence of nerve compression seem to be important factors in decision-making for treatment. Further studies are needed to identify subsets of patient which will most benefit from treatment.
\end{abstract}

Keywords: Stereotactic body radiation therapy - spinal bone metastasis

Asian Pac J Cancer Prev, 17 (3), 937-938

\section{Introduction}

Bone metastasis develops in approximately one-third of all patients with cancer. Bone metastases are important causes of morbidity and mortality in clinical practice and impair quality of life by causing pain, pathological fracture, spinal cord compression, bone marrow failure and sever hypercalcemia. Approximately, $70 \%$ of bone metastases involve vertebrates, as thoracic and lumbar levels being most common involvement sites. Although several methods are used in the treatment of bone metastases, radiotherapy (RT) is more commonly preferred method since it provides faster pain palliation that persists in long-term in most cases and has minimal adverse effects (Price et al., 1988; Chow et al., 2007; Murai et al., 2014).

Currently, RT is used for two distinct purposes as either palliative or stereotactic radiotherapy in case of oligometastasis and/or in patients with prolonged life expectation in lieu of surgical approaches (Price et al., 1988; Chow et al., 2007; Murai et al., 2014; De Bari et al., 2015) . The purpose of palliative therapy is to relieve complaints such as disease-related neurological deficits or pathological fractures; to improve local control; to prolong life expectation and time to progression; and to improve or maintain quality of life. Thus, therapeutic approaches with minimal toxicity that affect quality of life in minimal manner and provide long-term palliation are preferred. RT achieves improvement in pain control in more than $65 \%$ of cases and re-calcification is observed in the areas with bone destruction on radiographs obtained a few months after treatment. There is no consensus on dose and fraction of palliative radiotherapy and many studies have been conducted to compare total dose and fraction (e.g. 1x8 Gy, 3x10 Gy, 4x5 Gy). No difference was detected between longer and shorter therapies in any of the randomized studies including larger series (Price et al., 1988; Chow et al., 2007; Gerszten et al., 2007; Park et al., 2015). In a systematic review by Chow et al., it was reported that the most commonly used RT dose is $30 \mathrm{~Gy}$ given in 10 fractions and that partial pain response is 50$80 \%$ while complete pain response is $33 \%$ with palliative RT. Again, in the same analysis, it was reported that there is no difference between single- and multi-fraction dose regimens; however, need for repeated radiotherapy is more common in single-fraction regimens (Chow et al., 2007).

\section{Stereotactic Body Radiation Therapy (SBRT)}

In recent years, stereotactic radiosurgery is one of the most intensively investigated areas in spinal bone metastases. Stereotactic radiotherapy can be used in various regions of body by non-invasive stabilization methods and ability to obtain target images during therapy. Stereotactic radiotherapy can be applied to many primary and secondary cancers such as lung, liver, prostate and pancreas cancers or bone metastasis and various regions. Spinal SBRT defines giving high dose radiation to a target volume labeled stereotactically as either single or fractioned doses. It provides greater advantage in delivering dose, particularly in small target volume, when compared to other radiotherapy techniques. The highest dose is delivered to target volume while sharp reductions in the dose can be achieved in adjacent tissues and maximum protection is provided to surrounding vulnerable tissues such as medulla spinalis (Gerszten et al., 2007; Murai et al., 2014; Park et al., 2015).

In spinal bone metastases, total dose and number of fractions in SBRT are adjusted according to clinical condition, tumor type, previous history of RT, history of surgery, number of vertebrate involved and invasion of neural structures. Tumor progression occurs in epidural space when total dose is low while there is risk for 
radiation myelopathy when total dose is high in SBRT (Gerszten et al., 2007; Germano et al., 2015; Park et al., 2015).

There is no consensus on total dose and fraction scheme in SBRT and doses include 16-24 Gy in single fraction and 30-35 Gy/5 fractions, 24 Gy/2 fractions and 24-27 Gy/3 fractions in hypo-fractioned regimens. As yet there is no evidence support one regimen over another (Chow et al., 2007; Gerszten et al., 2007; Murai et al., 2014; De Bari et al., 2015; Germano et al., 2015; Moussazadeh et al., 2015; Park et al., 2015). Gerszten et al. delivered single dose SBRT to 500 lesions of 393 patients with spinal metastasis of which EBRT (30 Gy/10 fractions, $35 \mathrm{~Gy} / 15$ fractions) was given previously to 344 lesion. In that study, the most common primary indication was pain; mean intratumoral dose was 20 Gy; median follow-up was 21 months; and SBRT was most frequently delivered to thoracic region. Authors reported that success rate in long-term pain control was $96 \%$ in breast cancer and melanoma, $94 \%$ in renal cell cancer, $93 \%$ in lung cancer and $86 \%$ in all cancers while local control rate was $100 \%$ in breast, lung and renal cell carcinoma, $75 \%$ in melanoma and $90 \%$ in all cancers (Gerszten et al., 2007). Mantel et al. evaluated long-term outcomes in 32 patients with spinal metastasis received fractioned SBRT. SBRT was delivered at a median dose of $60 \mathrm{~Gy}$ in median 20 fractions. No grade 3 or 4 acute or chronic toxicity was observed during median followup of 20 months. Authors reported pain control of $61 \%$, neurological symptom control of $86 \%$ and 1- and 2-years local control rates of $92 \%$ and $84 \%$, respectively (Mantel et al., 2014). In a review by De Bari et al., it was reported that single dose spinal SBRT is effective and safe with long-term symptomatic response in radiation-resistant tumors such as renal cell carcinoma and melanoma (De Bari et al., 2015).

In SBRT, indication, technique, dose restrictions in critical organs and doses are clear. There is insufficient data on SBRT in de novo, retreatment and postoperative spine although 1-year local control rate has been reported as approximately $80 \%$. In spinal metastasis, it is important to pay attention in second series of irradiation, particularly regarding tolerance of spinal canal. It was reported that vertebral compression fractures after SBRT given due to spinal metastasis are more frequently seen in single dose RT regimens. Germano et al. reported that single dose SBRT is identical to multi-fraction SBRT regarding pain control, vertebral compression fracture and radiological appearance. Authors emphasized that surgery should be recommended, when there is progression in vertebral compression fracture, or if it becomes symptomatic after SBRT (Germano et al., 2015). In a study on patients received 278 high-dose and single-fraction (24 Gy) spinal SBRTs, Moussazadeh et al. investigated 5-years local tumor control and toxicity risk. Authors found that 5-years survival was $58 \%$ in radiation-resistant metastases, $22 \%$ in radiation-sensitive metastases and $19 \%$ in primary bone tumors. They also reported mild neuropathy and dermatitis by $22 \%$, myalgia/myositis by $8.3 \%$ and gastrointestinal findings by $5.6 \%$. Single-fraction spinal stereotactic radiosurgery retained an excellent safety profile among long-term survivors (>5 years) (Moussazadeh et al., 2015). In a study on 40 patients received spinal SBRT, Thibault et al. used SBRT at a dose of $30 \mathrm{~Gy}$ in 4 fractions as salvage therapy to the patients with local tumor progression. Authors found 1-year local control rate of $81 \%$ and 1-year overall survival rate of $49 \%$, reporting that SBRT is effective and feasible in salvage therapy (Thibault et al., 2015).

In conclusion, SBRT appears an effective and safe treatment modality in spinal bone metastasis, which can enhance local control and improve quality of life. Life expectation, predicted fracture risk, localization, quality, size and number of metastasis and presence or absence of nerve compression seem to be important factors in decision-making for treatment. Further studies are needed to identify subset of patient which will benefit from treatment.

\section{References}

Chow E, Harris K, Fan G, et al (2007). Palliative radiotherapy trials for bone metastases: A systematic review.J Clin Oncol, 25, 1423-36.

De Bari B, Alongi F, Mortellaro G, et al (2015). Spinal metastases: Is stereotactic body radiation therapy supported by evidences? Crit Rev Oncol Hematol. [Epub ahead of print]

Germano IM, Carai A, Pawha P, et al (2015). Clinical outcome of vertebral compression fracture after single fraction spine radiosurgery for spinalmetastases. Clin Exp Metastasis. 2015 Nov 17. [Epub ahead of print]

Gerszten PC, Burton SA, Ozhasoglu C, et al (2007). Radiosurgery for spinal metastases: clinical experience in 500 cases from a single institution. Spine (Phila Pa 1976), 32, 193-9.

Mantel F, Glatz S, Toussaint A, et al (2014). Long-term safety and efficacy of fractionated stereotactic body radiation therapy for spinal metastases. Strahlenther Onkol, 90, 1141-8.

Moussazadeh N, Lis E, Katsoulakis E, et al (2015). Five-year outcomes of high-dose single-fraction spinal stereotactic radiosurgery. Int J Radiat Oncol Biol Phys, 93, 361-7.

Murai T, Murata R, Manabe Y, et al (2014). Intensity modulated stereotactic body radiation therapy for single or multiple vertebral metastases with spinal cord compression. Pract Radiat Oncol, 4, 231-7.

Park HJ, Kim HJ, Won JH, et al (2015). Stereotactic body radiotherapy (SBRT) for spinal metastases: who will benefit the most from SBRT? Technol Cancer Res Treat, 14, 159-67.

Price P, Hoskin PJ, Easton D, et al (1988). Low dose single fraction radiotherapy in the treatment of metastatic bone pain: A pilot study. Radiother Oncol, 12, 297-300.

Thibault I, Campbell M, Tseng CL, et al (2015). Salvage stereotactic body radiotherapy (SBRT) following in-field failure of initial SBRT for spinal metastases. Int J Radiat Oncol Biol Phys, 93, 353-60. 